\title{
Awareness About Influenza and the Influenza Vaccine: A Doctor Survey to Assess Reasons for Influenza Vaccine Hesitancy in India
}

\author{
Hrishi Logani, ${ }^{1,}$, Rajesh Kumar ${ }^{2}$ \\ ${ }^{1}$ Delhi Public School, Vasant Kunj, New Delhi, India \\ ${ }^{2}$ Department of Internal Medicine, Paras Hospitals, Gurugram, India \\ Email address: \\ loganihrishi@gmail.com (H. Logani), raj8esh@yahoo.co.in (R. Kumar) \\ ${ }^{*}$ Corresponding author
}

\section{To cite this article:}

Hrishi Logani, Rajesh Kumar. Awareness About Influenza and the Influenza Vaccine: A Doctor Survey to Assess Reasons for Influenza Vaccine Hesitancy in India. International Journal of Infectious Diseases and Therapy. Vol. 6, No. 3, 2021, pp. 83-89. doi: $10.11648 /$ j.jijidt.20210603.11

Received: June 29, 2021; Accepted: July 19, 2021; Published: August 2, 2021

\begin{abstract}
Background: Influenza is a disease caused by four types of viruses and causes annual outbreaks. An outbreak of a new type of Influenza virus (A/H1N1) was declared as a pandemic by the World Health Organization (WHO) in 2009. Recently, there has been a lot of focus on the development of the COVID-19 vaccine but the Influenza vaccine, which has been available for a long time and has proven efficacy, is not being optimally utilized in India. Objective: The study aimed at assessing the awareness and attitude about Influenza and its vaccine amongst Indian doctors so as to analyze reasons for Influenza vaccine hesitancy in India. Method: An online questionnaire-based survey was undertaken between September 2020 and December 2020. 1000 doctors with valid Indian medical graduation (Bachelor of Medicine and Bachelor of Surgery, MBBS) or above were invited to participate. Results: The survey revealed that most doctors believed in Influenza vaccination as the best strategy to prevent infection, but lacked adequate awareness about the common facts regarding the appropriate season, diseases covered and the requirement of the influenza vaccine during pregnancy. Doctors opined that lack of patient awareness, casual counseling, few prescriptions by physicians, misconceptions about safety, and high cost of Influenza vaccine were the key reasons for vaccine hesitancy in patients. Conclusion: There is incomplete awareness amongst doctors regarding Influenza vaccination which might be a roadblock in the widespread acceptance of Influenza vaccines in India. Moreover, there is a need for patient-to-patient education through social media and the deployment of digital tools to address vaccine hesitancy in the country.
\end{abstract}

Keywords: Influenza, Vaccine, Hesitancy, Doctor-survey, India

\section{Introduction}

Influenza is a disease caused by four types of viruses namely Type A, Type B, Type C, and Type D. Its symptoms include mild to high-grade fever, running nose, sore throat, muscle and joint pain, headache, cough, and fatigue [1]. Complications of Influenza include viral pneumonia, secondary bacterial pneumonia, sinus infections, and worsening of health problems like asthma or coronary failure [2]. In 2019, the World Health Organization (WHO) reported that the Influenza virus caused 3 to 5 million severe illnesses and 290,000 to 650,000 deaths globally [1].

From history, it is evident that the Influenza virus spreads in yearly outbreaks. As many as three Influenza pandemics have occurred in the past century: The Spanish Influenza (also known as the Spanish Flu) in 1918 (17-100 million deaths), Asian Influenza in 1957 (two million deaths), and Hong Kong Influenza in 1968 (one million deaths) [3-5]. WHO declared an outbreak of a new type of Influenza A/H1N1 to be a pandemic in June 2009 [6] following which, India saw an increase in the number of these viral infections in 2012-13, and it has been worsening since December 2014; the death rate in India was over $6 \%$ which was significantly higher than the global death rate of $0.02 \%$ in 2009 [7]. Hospitals and clinicians reported much more severe Influenza disease than they had seen previously and confirmed very high mortality 
rates [8]. Also, a study in 2013 reported that each year around 127,092 people die in India due to Influenza-associated respiratory and circulatory diseases [9].

WHO recommends the Influenza vaccine for high-risk groups such as pregnant women, children aged less than five years, the elderly, healthcare workers, and people who have chronic illnesses such as HIV/AIDS, asthma, diabetes, heart disease, or are immuno-compromised among others $[10,11]$.

A meta-analysis done over the Western world found that fear of adverse reactions, lack of concern, efficacy concerns, and apprehensions about contraindications are the main causes for non-compliance [12]. A survey from the US shows that doctors were hesitant to prescribe the Influenza vaccine [13]. In a survey undertaken in China amongst healthcare workers, it was found that the primary reason for ignoring vaccination was due to the belief they were strong enough to not require immunization [14]. In 2016, a study undertaken amongst healthcare workers in Saudi Arabia found that safety concerns are a major barrier to the vaccination of health care professionals (HCPs) and misconceptions such as Influenza vaccine leading to Influenza disease and incorrect perceptions about the symptoms of Influenza in adults, were found [15]. Also, a survey of General Practitioners (GPs) from Italy revealed that the uptake of Influenza vaccine should be increased; however, one-tenth of GPs disagreed with the statement that vaccinating healthcare workers is crucial to prevent and control infections, and one fifth had not been vaccinated in the prior decade [16].

There are limited studies done to understand the reasons for Influenza vaccine hesitancy in India. Earlier surveys have been done on health care workers from 2-3 hospitals [17, 18]. Doctors play a crucial role in society in spreading education regarding transmission, prevention, and treatment of a disease.

The studies conducted amongst doctors so far report that there is limited awareness and vaccine coverage in India; the reasons for Influenza vaccine hesitancy include ignorance about vaccine availability, skepticism about efficacy, busy schedule, fear of side effects, and a misconception of not being at risk [15, 18]. The studies have shown that doctors were less likely to prescribe the Influenza vaccine even after the 2009 pandemic $[17,18]$. Therefore, more information is required regarding the awareness and attitude of doctors regarding Influenza, to identify the lacunae and specifically address them strategically.

\section{Material and Methods}

A study questionnaire was designed in consultation with a few physicians of good repute. The questionnaire was formatted in a Google Form ${ }^{\mathrm{TM}}$ and circulated to around 1000 doctors via an electronic medium (WhatsApp ${ }^{\mathrm{TM}}$ ) over 3 months starting September 2020 till December 2020. Multiple reminders were sent through WhatsApp ${ }^{\mathrm{TM}}$ communication and personal telephonic calls to some of them for completing the survey. The minimum qualification for taking the survey was Bachelor of Medicine and Bachelor of Surgery (MBBS) duly recognized by the Medical Council of India (MCI) and recording a valid Indian medical registration number was a mandatory requirement to proceed further. No financial remuneration was offered to the doctors for taking the survey, and appropriate consent was taken for publishing the data thus accrued in a scientific publication.

\subsection{Study Conduct and Data Collection}

The questionnaire was sent to doctors across all specialties, super-specialties, public and private hospitals, clinics, teaching, and non-teaching hospitals. This was done to reduce the bias and also to assess clinician awareness in general about Influenza and its vaccination irrespective of specialty in practice. None of the answers except email id, medical registration details, and consent was kept mandatory and the doctors were asked to respond comfortably as many questions as they consider relevant to their practice and omit the rest. The personal demographic details of doctors were obtained but were assured to be kept confidential.

The survey questionnaire sought information and opinion of the doctors regarding the following issues:

Efficacy of Influenza vaccination as a strategy to prevent community spread of infection.

Role of hand-washing vis-a-vis vaccination.

Some facts about Influenza vaccination.

Appropriate schedule and season.

Suitable age group.

Requirement and safety during pregnancy.

Diseases covered/prevented by vaccination.

Cost-effectiveness and availability.

Personal acceptance of Influenza vaccine by doctors.

Duration since they became aware of the Influenza vaccine. Use by self/family.

Doctors' practice regarding the prescription of Influenza vaccination.

Frequency.

Strategy.

Assessing and supplementing patient awareness.

Record-keeping (from prescription till actual vaccination).

Patient reminders for vaccination.

Doctors' opinion regarding various reasons for Influenza vaccine hesitancy amongst Indian patients and various strategies to improve vaccine compliance.

At the end of three months, 207 responses were received, out of which 28 responses were excluded where medical registration details could not be authenticated and 16 responses were excluded as they did not provide consent for sharing the data for publication. Finally, 163 authentic responses were available for analysis.

\subsection{Statistical Analysis}

The data collected from Google Form ${ }^{\mathrm{TM}}$ was subjected to statistical analysis using Microsoft Excel and Stata 16.0 software. An in-depth analysis of the data was done, focusing on doctors' prescription strategy and their belief in the Influenza vaccine. The responses of doctors of different ages (26-40 years and age $>40$ years) were also compared. A chi-square test was also performed to check the statistical significance of the data obtained. 


\subsection{Ethical Consideration}

Ethical consideration for this study was taken by the Ethics committee (ECR No. ECR/249/Inst./HAR/2013/RR-19 dated 08/28/2020), Paras Hospitals, Gurugram before starting the study.

\section{Results}

Table 1. Influenza vaccine as a strategy.

\begin{tabular}{|c|c|c|c|c|}
\hline Question & $\begin{array}{l}\text { Number of } \\
\text { Responses }\end{array}$ & $\begin{array}{l}\text { Agree } \\
(\%)\end{array}$ & $\begin{array}{l}\text { Disagree } \\
(\%)\end{array}$ & $\begin{array}{l}\text { Neutral } \\
(\%)\end{array}$ \\
\hline Vaccination is the best strategy to prevent Influenza infection & 163 & $76.9^{*}$ & 15.3 & 6.7 \\
\hline Hand-washing is more effective than the vaccine for the prevention of Influenza & 162 & 55.6 & $33.3^{*}$ & 11.1 \\
\hline The Influenza vaccine is helpful in the prevention and community transmission of the disease & 163 & $79.8^{*}$ & 12.3 & 8.0 \\
\hline The risk of side effects outweighs the benefits of the Influenza vaccine & 158 & 30.4 & $56.9^{*}$ & 12.7 \\
\hline $\begin{array}{l}\text { Mass vaccination with the Influenza vaccine will help reduce the disease burden on the health system in } \\
\text { India }\end{array}$ & 160 & $73.8^{*}$ & 17.5 & 8.8 \\
\hline *Correct Answer & & & & \\
\hline
\end{tabular}

The survey data (Table 1) reveals that more than $75 \%$ $(n=125)$ of the doctors agree that the Influenza vaccine is the best strategy to prevent infection, next to hand washing. Also, the majority $(73.8 \%, n=118)$ were convinced that it is helpful in the prevention of community transmission. More than $70 \%$ of the doctors agreed that mass vaccination will be helpful to reduce the disease burden. $30.4 \%(\mathrm{n}=48)$ believed that the risk of side effects outweighs the benefits of Influenza. $27.3 \%$ $(n=44)$ agreed that the Influenza vaccine is effective only in the young and old population.

Table 2. Facts about Influenza vaccine.

\begin{tabular}{|c|c|c|c|}
\hline Facts about Influenza Vaccine & Number of Responses & Correct (\%) & Incorrect (\%) \\
\hline Appropriate schedule & 162 & Annually (77.8) & $\begin{array}{l}22.2 \\
\text { Pre-winter: } 40.4\end{array}$ \\
\hline Appropriate season & 161 & Pre-monsoon \& Pre-winter (36.0) & $\begin{array}{l}\text { Pre-monsoon: } 21.7 \\
\text { Summer: } 1.9\end{array}$ \\
\hline Appropriate age group & 162 & Till one is alive (75.9) & 24.1 \\
\hline $\begin{array}{l}\text { Requirement and safety during } \\
\text { pregnancy }\end{array}$ & 146 & Any Trimester (26.7) & $\begin{array}{l}\text { Not required: } 27.4 \\
2^{\text {nd }} \text { Trimester: } 26.0 \\
3^{\text {rd }} \text { Trimester: } 19.9\end{array}$ \\
\hline Diseases covered/prevented & 155 & Swine-flu only (31.0) & $\begin{array}{l}\text { Both Bird-flu \& Swine flu: } 46.5 \\
\text { Bird flu only: } 0.6 \\
\text { Neither Bird-flu nor Swine flu: } 21.9\end{array}$ \\
\hline
\end{tabular}

The survey data (Table 2) also provides insight into the level of awareness of the doctors regarding the common facts about the Influenza vaccine. Most $(77.8 \%, n=126)$ of the doctors were aware of the annual requirement of the vaccine and its provision to any age group. However, only $36 \%(n=58)$ responded correctly regarding the season for administering the vaccine and only $31 \%(n=48)$ were aware of the diseases prevented by the vaccine. Most of the doctors agreed that a vaccine was required during pregnancy; however, only $26.7 \%$ $(n=39)$ responded correctly about the administration of the vaccine in any trimester during pregnancy.

It was found that doctors' age did not have a significant impact on their opinion regarding the Influenza vaccine $(\mathrm{p}=0.818)$. However, doctors' qualifications did have a significant impact on their perception; doctors who possessed only a graduate degree (MBBS) were less likely to consider vaccination as the best strategy than those with additional post-graduate qualifications ( $\mathrm{p}=0.038)$.

Survey data revealed some interesting insights about the prescription strategy of doctors.

(i) Frequency of prescription: About $48 \%(n=73)$ of doctors hardly ever prescribe the Influenza vaccine, $27 \%(\mathrm{n}=41)$ prescribe it to about a quarter of their patients, and about $16 \%$ $(n=25)$ to half or more of their patients.

(ii) Strategy of prescription: The strength of prescription strategy was categorized as follows:

Casual: doctors felt that it might be beneficial but did not specifically prescribe it.

Optional: doctors believed and informed the patient that it was beneficial but left the decision of getting vaccinated to the patient.

Strong: doctors strongly believed and informed the patient that it was beneficial and insisted upon getting vaccinated.

Mandatory: doctors had a strong conviction that it was beneficial and accordingly patient had no choice but to get vaccinated.

Out of 145 responses, most responses $(48.96 \%)$ were in the 'optional' category, $43.44 \%$ were in the 'strong' category, and $7.58 \%$ were in the 'casual' category. No responses were obtained in the 'mandatory' category.

(iii) Assessing and supplementing patient awareness: About $62 \%(n=95)$ of doctors report that less than $10 \%$ of their patients are already aware of the Influenza vaccine at the time of prescription, and $76 \%(n=114)$ of doctors feel that out of those patients who are already aware, less than $10 \%$ ask for it themselves. 
(iv) Record-keeping (from prescription till actual vaccination): Nearly $57 \%(\mathrm{n}=85)$ of doctors rarely keep any record of their vaccine prescriptions, while about $32 \%(n=48)$ of doctors do maintain a record of at least half of their prescriptions.

(v) Patient reminders for vaccination: Out of those doctors who maintain some kind of a record of their vaccine prescriptions, about $74 \%(\mathrm{n}=98)$ do not send any reminders to patients, while the rest send reminders through at least one of the modalities (text or WhatsApp ${ }^{\mathrm{TM}}$ messages, telephonic or app-based reminders).

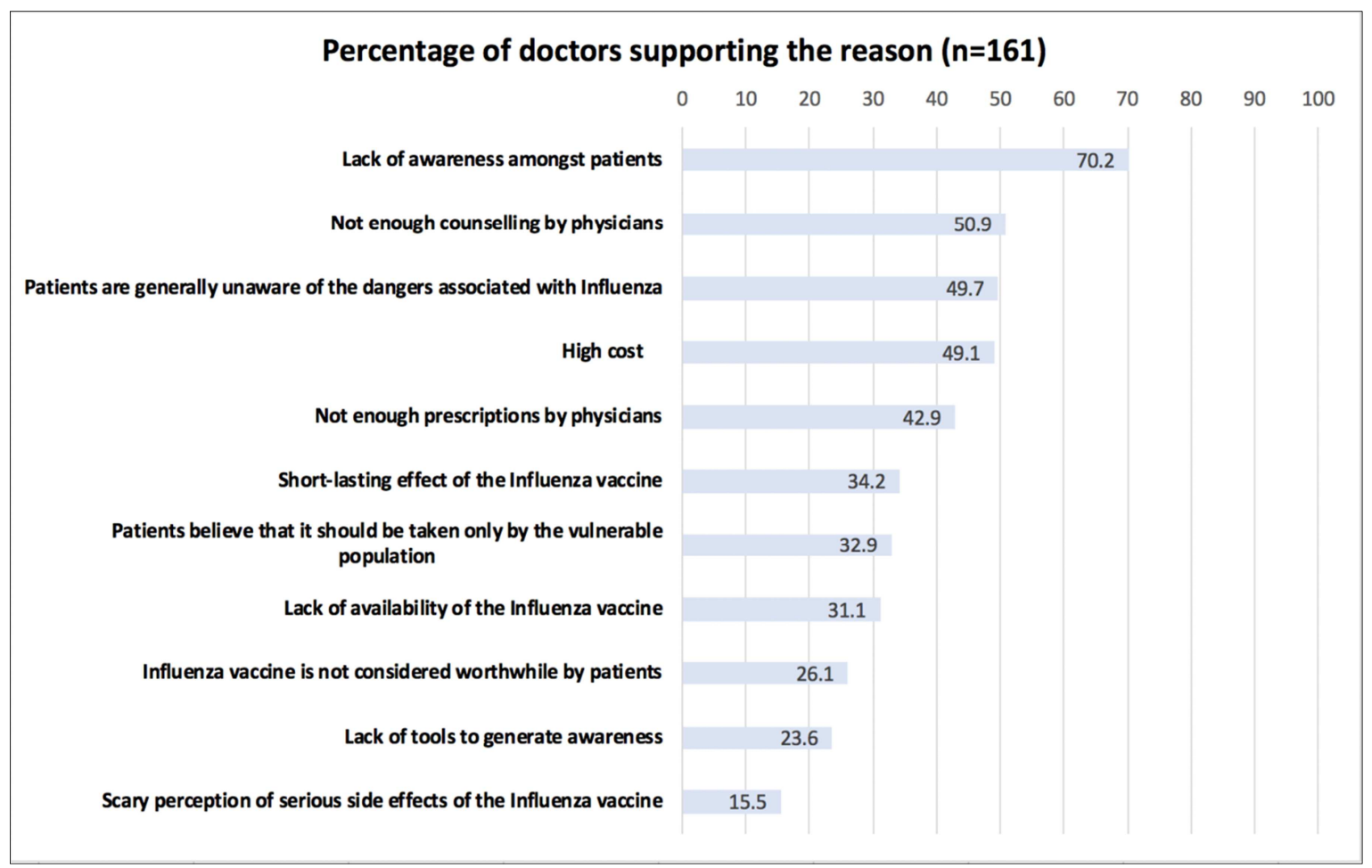

Figure 1. Doctors' opinion about reasons for Influenza vaccine hesitancy amongst Indian patients.

The survey also solicited doctors' opinions about the reasons for Influenza vaccine hesitancy amongst Indian patients (Figure 1). Most of the doctors $(70.20 \%)$ cited a lack of awareness amongst the patients as the major reason for hesitancy. Other factors reportedly contributing to vaccine hesitancy were inadequate counseling by the physicians, ignorance about the dangers of the disease, high cost, and few doctor prescriptions in that order.

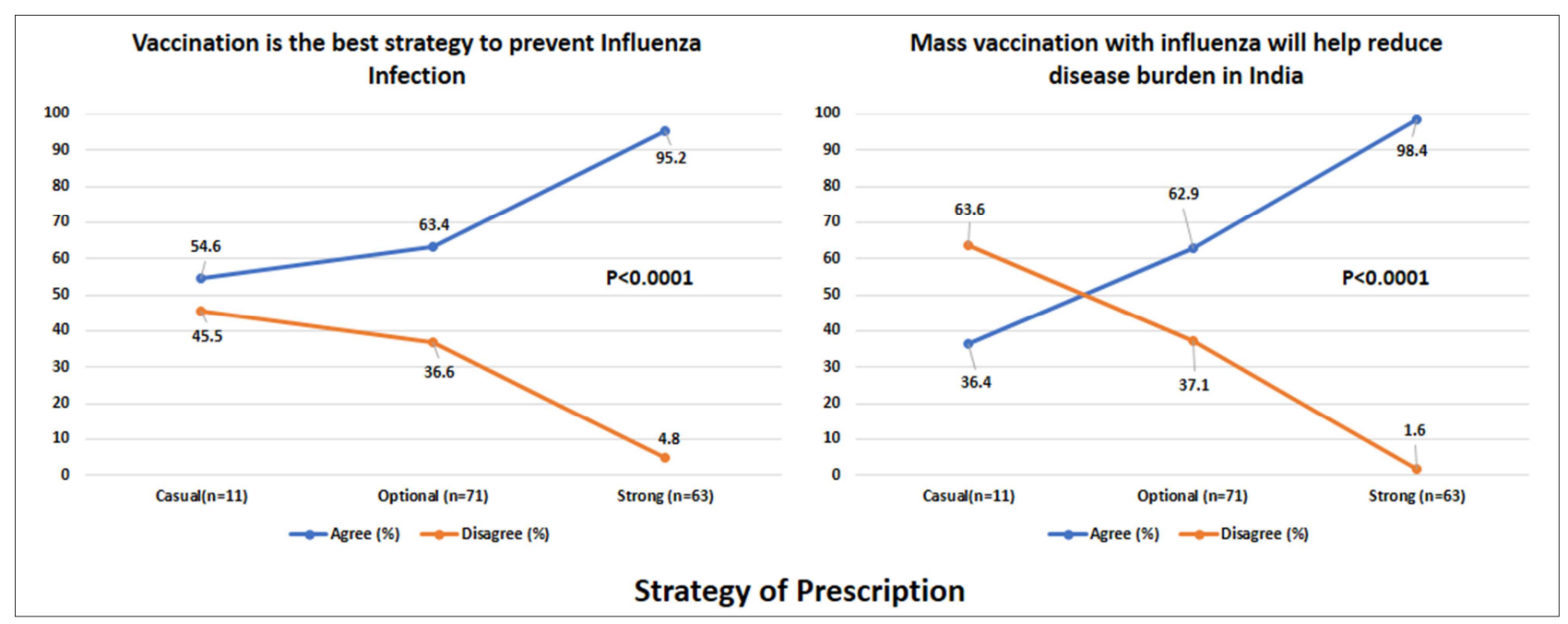

Figure 2. Doctors'belief in Influenza vaccine vs their prescription strategy.

A correlation was found between doctors' prescription strategy and their belief in the Influenza vaccine (Figure 2). It was found that the doctors who reported vaccination as the best strategy against infection and were in favor of mass vaccination were significantly more likely to have a stronger strategy of prescription of Influenza vaccine $(p<0.0001)$. 


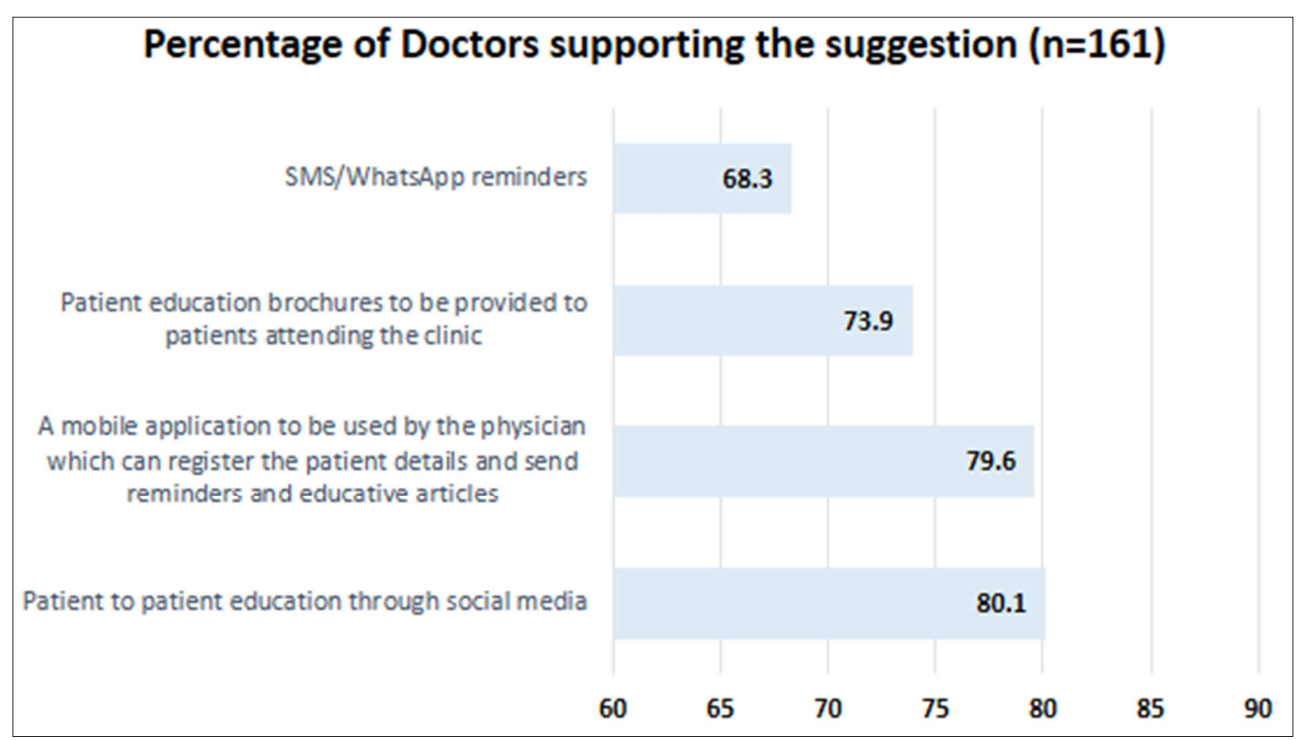

Figure 3. Doctors'suggestions to improve vaccine compliance in India.

Doctors' suggestions to improve vaccine compliance in India were also sought in the survey (Figure 3). They recommended patient-to-patient education through social media, using a mobile application, use of patient education brochures, and also suggested sending of the SMS/WhatsApp reminders.

\section{Discussion}

The data obtained from the survey reveals that more than $70 \%$ $(n=127)$ of the doctors agree that the Influenza vaccine is the best strategy to prevent infection (next to hand-washing), is helpful in prevention and community transmission of disease, and can reduce disease burden in India (Table 1). However, there is a lack of routine annual use of the Influenza vaccine in India. The findings suggest that the majority of the doctors are not aware of the appropriate season of Influenza vaccination in India, the requirement of the vaccine during pregnancy, and the diseases prevented by the Influenza vaccine (Table 2). An earlier study undertaken in pregnant women in north India has shown that the Influenza vaccine has not been prescribed during pregnancy and only $10 \%$ of obstetricians surveyed had themselves been vaccinated against the Influenza though $90 \%$ believed that Influenza could be dangerous [19]. Also, to our knowledge, this is the first survey in India to collect data from 163 doctors about their perception of Influenza vaccination during pregnancy. In this study, $72.6 \%(n=106)$ of the doctors reported that there is a need for an Influenza vaccine during pregnancy but only $26.7 \%(n=39)$ correctly reported that the vaccine can be given during any trimester. While the age of doctors did not have a significant impact on their opinion of the Influenza vaccine $(\mathrm{p}=0.818)$, a strong correlation was found between the level of doctor qualification and their awareness, acceptance, and likelihood of prescription of Influenza vaccine $(p=0.038)$. This suggests that overall awareness is needed, more so among graduate doctors.

The survey also collected data about personal perception and prescription of the Influenza vaccine by the doctors. About 62\% $(n=101)$ of doctors had been aware of the Influenza vaccine for over five years, about $80 \%(n=130)$ of doctors were convinced that the Influenza vaccine is helpful in the prevention and community transmission of disease in India and about $74 \%(\mathrm{n}=118)$ were in favor of mass vaccination in India, but it was observed that about $46 \%$ $(n=75)$ had never taken Influenza vaccine themselves and among the rest, about $41 \%(n=66)$ had taken it in the last $1-2$ years only. On similar lines, about $41 \%(n=66)$ had never got any family member vaccinated, and about $46 \%(n=75)$ had got one of the family members vaccinated in the past 1-2 years. This reflects a relatively casual approach towards adult vaccination by doctors.

The data from the survey also determines possible reasons (from the doctors' perspective) for the Influenza vaccine hesitancy in India in general (Figure 1). The top five reasons observed for the hesitancy were lack of awareness amongst patients, inadequate counseling by physicians, lack of awareness amongst patients regarding the severity of the disease, high cost, and short-lasting effect of the Influenza vaccine. This survey highlights that there is a need for spreading awareness both amongst doctors as well as the general population; it also indicates that the concept of adult immunization for Influenza needs major emphasis in India.

A strong positive correlation was found between the belief versus the prescription strategy (Figure 2). It is evident from the data that about $95.2 \%(\mathrm{n}=60)$ of doctors who strongly prescribe the Influenza vaccine agree that the Influenza vaccine is the best strategy to prevent the infection. This strength of agreement decreased to $63.4 \%(n=45)$ for casual prescribers and $54.6 \%(\mathrm{n}=6)$ for optional prescribers. The trend was statistically significant $(p=0.000016)$. Thus it may be inferred that a stronger belief in the Influenza vaccine as a strategy could positively influence the strength of prescription. Similarly, $98.4 \%(n=61)$ of strong prescribers agreed that mass vaccination could reduce the disease burden on the healthcare 
system in India, while only $36.4 \%(n=4)$ of casual prescribers felt so $(p=0.000000057)$.

The survey also collected doctors' suggestions for improving Influenza vaccine compliance in India. Most of the doctors believed patient-to-patient education through social media is the best way to spread awareness, followed by a web-based application that can automatically send a reminder to patients for vaccination (Figure 3 ). It is observed that the creation of a database/patient registry creates a significant impact on mass immunization. For instance, a population database has been created by the Government of India using the CoWIN ${ }^{\mathrm{TM}}$ application for mass vaccination against COVID-19; this database seems to be making a significant impact on vaccination, and a similar strategy needs to be adopted to increase the acceptance of Influenza vaccine both amongst doctors as well as in the general population. Many earlier studies have endorsed the need for such tools for Influenza vaccination $[20,21]$.

\section{Limitations}

The responses were self-reported by doctors, not subject to independent verification, and potentially influenced by common perceptions. The sample size of the study is small, so the findings cannot be generalized. Despite its limitations, the study adds to the understanding of the influenza vaccine hesitancy.

\section{Conclusion}

The findings generated from this survey provide a good insight into doctors' opinions regarding the Influenza vaccine; it is observed that there is a general lack of clarity in the understanding of Influenza and its vaccine amongst Indian doctors. There is a need for enhancing awareness amongst doctors regarding the Influenza vaccine: its benefits, side-effects, appropriate season of administration, its role during pregnancy, and diseases prevented by the vaccine. Interestingly, the level of personal knowledge and conviction of the doctors about the utility of Influenza vaccination has a strong positive correlation with the likelihood and strength of their prescription. Doctors with a strong prescription strategy also support mass vaccination for the prevention of community transmission. Doctors believe that limited awareness amongst the general population, lack of strong prescriptions and counseling by doctors, and the repetitive need for annual vaccination are additional roadblocks to the widespread acceptance of the Influenza vaccine in India. Doctors suggest that patient-to-patient education through social media and utilizing digital tools in vaccination programs coupled with mass media support could significantly improve awareness about the Influenza vaccine and its uptake in India.

\section{Acknowledgements}

The authors hereby acknowledge Dr Sunita Taneja and Dr
Kiran Bhatia, Centre for Health Research and Development, for their valuable inputs and statistical analysis.

\section{References}

[1] Influenza (Seasonal) [Internet]. World Health Organization. 2018 [cited 2021 May 12]. Available from: https://www.who.int/news-room/fact-sheets/detail/influenza-(s easonal).

[2] Longo DL. Influenza. In: Harrison's principles of internal medicine. 20th ed. New York: McGraw-Hill; 2018.

[3] Spreeuwenberg P, Kroneman M, Paget J. Reassessing the Global Mortality Burden of the 1918 Influenza Pandemic. Am J Epidemiol. 2018 Dec 1; 187 (12): 2561-2567. doi: 10.1093/aje/kwy191. PMID: 30202996; PMCID: PMC7314216.

[4] World Health Organization. Ten things you need to know about pandemic influenza (update of 14 October 2005). Wkly Epidemiol Rec. 2005 Dec 9; 80 (49-50): 428-31. English, French. PMID: 16372665.

[5] Jilani TN, Jamil RT, Siddiqui AH. H1N1 Influenza. 2020 Nov 30. In: StatPearls [Internet]. Treasure Island (FL): StatPearls Publishing; 2021 Jan-. PMID: 30020613.

[6] Chan M. World now at the start of 2009 influenza pandemic [Internet]. 2009 [cited 2021 May 12]. Available from: https://www.who.int/mediacentre/news/statements/2009/h1n 1 pandemic_phase6_20090611/en/\#: :text=The\%20world\% 20 is $\% 20$ now $\% 20$ at, right $\% 20$ at $\% 20$ the $\% 20$ very\%20beginnin g.

[7] Chadha MS, Hirve S, Dawood FS, Lele P, Deoshatwar A, Sambhudas $\mathrm{S}$ et al. Burden of seasonal and pandemic influenza-associated hospitalization during and after 2009 A (H1N1) pdm09 pandemic in a rural community in India. PLoS One. 2013 May 15; 8 (5): e55918. doi: 10.1371/journal.pone.0055918. PMID: 23690913; PMCID: PMC3654970.

[8] Bui C, Narasimhan P, MacIntyre R. Infectious Disease Outbreaks in India- Challenges and opportunities [Internet]. School of Population Health. 2016 [cited 2021 May 11]. Available from: https://sph.med.unsw.edu.au/infectious-diseases-blog/infectiou s-disease-outbreaks-india-challenges-and-opportunities.

[9] Narayan VV, Iuliano AD, Roguski K, Bhardwaj R, Chadha M, Saha $\mathrm{S}$ et al. Burden of influenza-associated respiratory and circulatory mortality in India, 2010-2013. J Glob Health. 2020 Jun; 10 (1): 010402. doi: 10.7189/jogh.10.010402. PMID: 32373326; PMCID: PMC7182391.

[10] Vaccine use [Internet]. World Health Organization. 2020 [cited 2021 May 12]. Available from: https://www.who.int/influenza/vaccines/use/en/.

[11] Vaccines against influenza WHO position paper - November 2012. Wkly Epidemiol Rec. 2012 Nov 23; 87 (47): 461-76. English, French. PMID: 23210147.

[12] Hollmeyer HG, Hayden F, Poland G, Buchholz U. Influenza vaccination of health care workers in hospitals--a review of studies on attitudes and predictors. Vaccine. 2009 Jun 19; 27 (30): 3935-44. doi: 10.1016/j.vaccine.2009.03.056. Epub 2009 Apr 8. PMID: 19467744. 
[13] Davis MM, McMahon SR, Santoli JM, Schwartz B, Clark SJ. A national survey of physician practices regarding influenza vaccine. J Gen Intern Med. 2002 Sep; 17 (9): 670-6. doi: 10.1046/j.1525-1497.2002.11040.x. PMID: 12220362; PMCID: PMC1495108.

[14] Ma Y, Li T, Chen W, Chen J, Li M, Yang Z. Knowledge, Attitudes and Practices (KAP) toward seasonal influenza vaccine among young workers in South China. Hum Vaccin Immunother. 2018 May 4; 14 (5): 1283-1293. doi: 10.1080/21645515.2017.1423157. Epub 2018 Feb 12. PMID: 29308971; PMCID: PMC5989888.

[15] Alshammari TM, Yusuff KB, Aziz MM, Subaie GM. Healthcare professionals' knowledge, attitude and acceptance of influenza vaccination in Saudi Arabia: a multicenter cross-sectional study. BMC Health Serv Res. 2019 Apr 15; 19 (1): 229. doi: 10.1186/s12913-019-4054-9. PMID: 30992004; PMCID: PMC6469139.

[16] Levi M, Bonanni P, Biffino M, Conversano M, Corongiu M, Morato P et al. Influenza vaccination 2014-2015: Results of a survey conducted among general practitioners in Italy. Hum Vaccin Immunother. 2018 Jun 3; 14 (6): 1342-1350. doi: 10.1080/21645515.2018.1430543. Epub 2018 Mar 30. PMID: 29451834; PMCID: PMC6037480.

[17] Bali NK, Ashraf M, Ahmad F, Khan UH, Widdowson MA, Lal $\mathrm{RB}$ et al. Knowledge, attitude, and practices about the seasonal influenza vaccination among healthcare workers in Srinagar, India. Influenza Other Respir Viruses. 2013 Jul; 7 (4): 540-5. doi: 10.1111/j.1750-2659.2012.00416.x. Epub 2012 Aug 2. PMID: 22862774; PMCID: PMC5781001.

[18] Hadaye RS, Manapurath RM, Gadapani BP. Awareness and acceptance of H1N1 vaccination among physicians: Experience of 2017 vaccination campaign. J Educ Health Promot. 2019 Apr 24; 8: 82. doi: 10.4103/jehp.jehp_356_18. PMID: $31143799 ;$ PMCID: PMC6512216.

[19] Koul PA, Bali NK, Ali S, Ahmad SJ, Bhat MA, Mir H et al. Poor uptake of influenza vaccination in pregnancy in northern India. Int J Gynaecol Obstet. 2014 Dec; 127 (3): 234-7. doi: 10.1016/j.ijgo.2014.05.021. Epub 2014 Jul 17. PMID: 25085688 .

[20] Lindley MC, Horlick GA, Shefer AM, Shaw FE, Gorji M. Assessing state immunization requirements for healthcare workers and patients. Am J Prev Med. 2007 Jun; 32 (6): 459-65. doi: 10.1016/j.amepre.2007.02.009. PMID: 17533060.

[21] Canning HS, Phillips J, Allsup S. Health care worker beliefs about influenza vaccine and reasons for non-vaccination--a cross-sectional survey. J Clin Nurs. 2005 Sep; 14 (8): 922-5. doi: 10.1111/j.1365-2702.2005.01190.x. PMID: 16102143. 